\title{
Téoros
}

Revue de recherche en tourisme

\section{« Nos » touristes depuis 20 ans}

\section{Jacques Demers}

Volume 4, numéro 2, juillet 1985

Les clientèles touristiques : un tour d'horizon

URI : https://id.erudit.org/iderudit/1080624ar

DOI : https://doi.org/10.7202/1080624ar

Aller au sommaire du numéro

Éditeur(s)

Université du Québec à Montréal

ISSN

0712-8657 (imprimé)

1923-2705 (numérique)

Découvrir la revue

Citer cet article

Demers, J. (1985). « Nos » touristes depuis 20 ans. Téoros, 4(2), 2-5.

https://doi.org/10.7202/1080624ar d'utilisation que vous pouvez consulter en ligne.

https://apropos.erudit.org/fr/usagers/politique-dutilisation/ 


\title{
«Nos» touristes depuis 20 ans
}

\author{
par Jacques Demers*
}

Traiter des touristes depuis les vingt dernières années moblige à reculer dans le temps. A cet égard, je dois prévenir le lecteur, ceci me rend nostalgique et rêveur; dans ces circonstances, j'ai plutồt le goût de me transformer en poète et de foutre en /air les dossiers statistiques que jai accumulés depuis.

Sans doute, les éditeurs de Téoros me pardonneront si je me laisse bercer par les souvenir d'antan. Sans pour autant atténuer la teneur académique de cette revue, je veux d'abord revive ces souvenirs avant de passer à l'austéritế analytique de chiffres. En termes doctoraux, on parle alors de revue historique des événements.

L'histoire commence donc il y a vingt ans, en 1965. Le ministere du Tourisme de la Chasse et de la Pêche, venait a peine de naitre alors que le touriste, lui, existait dêjâ depuis longtemps. On venait de découvrir son importance, d'autant plus que l'Exposition Universelle de 1967 approchait. Encore loin de la panique de Québec 1534-1984, nous vivions tout au moins une espèce d'euphorie attisée par Gabriel Loubier, alors ministre du Tourisme.

A cette époque, il $\mathrm{y}$ avait beaucoup de touristes et l'activité était telle qu'il ne restait plus de temps pour songer à maintenir cet élan. On le croyait perpétuel; on s'imaginait que l'accroissement annuel de 7 a $10 \%$ de touristes ne s'arreterait jamais. Dans ces circonstances, à quoi bon s'attarder à des analyses de marché ou à des études de comportement alors que notre énergie suffisaît à peine à développer tout le potentiel. Conséquemment, on connaissait peu nos touristes.

Or, apres l'Expo 67, on ressentit un apaisement des activités touristiques sans pour autant voir diminuer le nombre de visiteurs au Québec. Cependant, de plus

- Jacques Demers est president de linstitut nord américain de recherche an tourisme inc. en plus on ressentait le besoin de mieux connailtre celui qui nous rendait visite. On songeait alors aux non-résidents (le touriste résident nous préoccupait peu à ce moment lâl compte tenu de leur grand nombre. C'est ainsi qu'en 1968 on créa le service de la recherche socio-économique au MTCP. Son mandat consistait à analyser qualitativement et quantitativement les touristes. De plus, le service, représenté par son directeur, devait siéger sur un comité technique que venaient tout juste de créer les ministres du Tourisme en conférence fédérale-provinciale. Ce comitét"l avait pour fonction de mettre au point des enquetes pan-canadiennes afin de créer une banque de données sur l'ensemble des touristes au Canada. C'est de lả d'ailleurs qu'originent quelques-unes des etudes statistiques actuellement disponibles.

Ne voulant pas être en reste, cette mème année, le service de la recherche réalise sa propre enquễte aux frontières. C'étaít une première et malgré les lacunes méthodologiques a l'époque, par ce moyen, nous pouvions disposer alors de quelques informations qualitatives sur nos touristes. Ces enquêtes se sont d'ailleurs répétées en 1970 et en 1975. Apres cette date, elles ont été abandonnées. D'ailleurs, elles ne touchaient que l'automobiliste voyageant au cours des mois d'été. Bien que ceux-ci représentent la part la plus substantielle des touristes, ces données demeuraient partielles.

C'est ainsi que de nouvelles avenues plus scientifiques ont été dorénavant exploitées. A ce propos, le lecteur voudra bien se référer à l'article signé par Patrick Cluzeau quil explique avec plus de détails les enquêtes actuellement disponibles. Du même coup, ou verra si ce n'est déja fait, les différentes notions entre visites/personnes, visites/provinces, voyages/personnes entrees aux frontieres.

Ce bref rappel historique, bien qu'il me rende nostalgique, me fait aussi réfléchir. II est impensable que dans un contexte économique comme le nỏtre on ait tant tardé à se doter d'une base statistique stable en matière touristique. La rédaction de cet article me place brutalement davant une situation, alors que je n'ai pu véritáblement trouver des séries statistiques valables afin de suivre l'évolution d'une de nos principales industries du Québec. On peut bien rappeler des faits, des événe. ments mais bien malin celui qui peut expliquer à partir des données précises, l'évo" lution en termes qualitatifs de nos touristes depuis vingt ans. Bien que l'étude des voyages entre le Canada, les Etats-Unis et les autres pays existe depuis plus de 30 ans, elle ne nous informe que sur le nombre de voyages et ne foumit que peu de données qualitatives. L'Enquête sur les voyages des Canadiens est encore trop récente, dans sa structure actuelle, et ne fournit pas assez de recul pour les fins du présent article. Par contre, les études poursuivies par Travel Data existent depuis 1966 et ont une constance en termes de base statistique. Cependant. ces études se limitent aux voyages des Canadiens et ne touchent aucunement aux Américains qui viennent au Québec. De plus, elles s'adressent aux touristes d'agrément et négligent les autres formes de touristes.

Voild donc une situation fort embarassante pour celui qui désire poursuivre une analyse evolutive des touristes au cours des deux dernieres décennies. Je ne peux que deplorer cette situation.

Malgré cette contrainte, j'ai pu brosser un tableau très sommaire de quelques caractéristiques permettant d'indiquer des tendances à partir des études de Travel Data, en prenant pour acquis que les tendances manifestées par les Canadiens sont un indicateur d'un comportement normal des touristes en général. On peut le comparer à un indicateur boursier, qui en valeur $a b=$ solue n'a relativement pas de signification, sauf qu'il permet de comparer une situation par rapport á une autre.

Pour les fins de l'analyse, celle-ci se situera entre 1966 et 1981 , et je ferai référence à un certain nombre de tableaux extraits des études de Travel Data. 
Le premier tableau nous indique un certain nombre d'aspects. D'abord, on remarque, málgré la récession des dernières années, que les Canadiens ont néanmoins continué à faire des voyages de vacances. Or, selon les informations dont nous disposons, cette tendance s'est maintenue aussi à l'intérieur du Canada. De 1970 a 1981, on note egalement une stabilité dans le comportement des vacanciers. Plus de la moitié de la population fait au moins un voyage par année alors qu'une faible proportion des vacanciers demeurent à la maison. Nous pouvons présumer que l'autre moitié ne prend pas de vacances pour une raison ou une autre.

Dans le tableau suivant on perçoit une certaine évolution :

De 1966 a 1979 , il y eut une lente progression vers la réalisation de vovages multiples alors que par la suite la situation semble se stabiliser. II en résulte que les vacanciers ayant fait deux voyages et plus par année ont presque doublé au cours d'une période de 13 ans pour passer de $10 \%$ à $19 \%$. Par contre, la propertion de ceux qui préfèrent réaliser un seul wowage est parfaitement inchangée tout au long de cette meme période. En fait, cette modification de comportement est due au plus grand nombre de vacanciers qui peuvent se permettre de faire des voyages de vacances. Cette tendance se réflète dans la partie inférieure du tableau oú $54 \%$ des adultes restaient à la maison en 1966 , alors que ce taux est passé a $45 \%$ depuis.

La façon de se déplacer, on le devine, a été fortement influencée par nos amis de I'OPEP. Les coûts de l'essence ont largement favorisé l'utilisation de l'avion et sans doute, les Canadiens attirés vers le sud ont davantage préféré laisser la voiture à la maison. C'est ainsi que de
1966 a aujourd'hui, l'avion est trois fois plus utilisé comme mode de transport des touristes. Le tableau 3 est explicite à ce sujet.

On peut se demander si cette tendance se maintiendra. J'ail l'impression pour ma part qu'elle s'acoroitra à cause de la nouvelle déréglementation dans le secteur du transport aérien. Cependant, I'automobile conservera toujours une place de choix d'autant plus que les exigences du gouvernement américain vis-à-vis les constructeurs automobiles imposent un accroisement du kilométrage au litre d'essence.

Le fait que l'avion acquiert plus d'adeptes, on peut se demander si l'agent de vovage est davantage sollicité. La corrélation existe. Entre 1970 , soit au début de l'accroissement des prix de l'énergie, et 1978, l'agence de voyage a triplé son marché pour atteindre une stabilité relative dans le cas des voyages au Canada et aux États. Unis. (Tableau 4)

L'accroissement pour le marché vers l'Europe fut cependant moins marqué quoiqu'il s'est accru sensiblement. Dans ce dernier cas, bien sûr, l'automobile ne peut être utilisée; par contre ceci nous indique que d'autres facteurs, outre le coût de l'essence, sont responsables des changements de comportements.

Le moment oủ l'on prend ses vacances a aussi subi des modifications évidentes au cours des deux dernières décennies. Le tableau 5 nous montre les variations saisonnières.

Bien que l'été demeurera toujours la saison idéale des vacances, on ne peut demeurer insensible au fait que depuis les vingt dernières années, l'étalement saisonnier s'est légèrement modifié. On peut croire qu'un produit mieux adapté aurait pour effet d'accentuer davantage cette attitude. II aurait êté intéressant d'établir à ce propos un certain nombre de corrélations afin de vérifier diverses hypothèses. Par exemple, en établissant la relation entre la saison et la destination, peut-être découvrirait-on que plus de gens s'envolent vers le sud au printemps ou encore que plus de vacanciers fractionnent leurs périodes de vacances au cours d'une année. Pour une analyse plus poussée, il faudrait sans doute effectuer une commande spéciale auprés de Trave Data, Cependant, une observation rapide du prochain tableau, recoupé avec les tableaux 2 et 5, nous confirme que le touriste a davantage tendance à faire plus d'un voyage au cours d'une måme année.

On remarque au tableau 6 que le nombre de nuitées a peu diminué entre la période observée, pour les voyages au Canada et aux Etats-Unis alors qu'outre-mer la varia. tion est plus évidente. Ces dernieres fluc. tuations correspondent ordinairement aux variations des taux de change et aux coùts du transport. On sait d'ailleurs que l'un et l'autre sont de plus en plus fluc. tuants.

Enfin, un dernier point a attiré notre attention (études de Travel Data). II s'agit du goût de plus en plus prononce qu'ont les touristes à séjoumer au mëme endroit. On signale un accroissement de cette habitude de plus de $10 \%$.

Ces quelques observations, bien que très sommaires, permettent quand même de tirer quelques conclusions. Au préalable, je tiens cependant à rappeler les limites des données utilisées. II serait présomptueux de trancher systématiquement la question compte tenu de la disparité des données disponibles. Je m'en suis servi. tout au plus comme indicateur et en ce

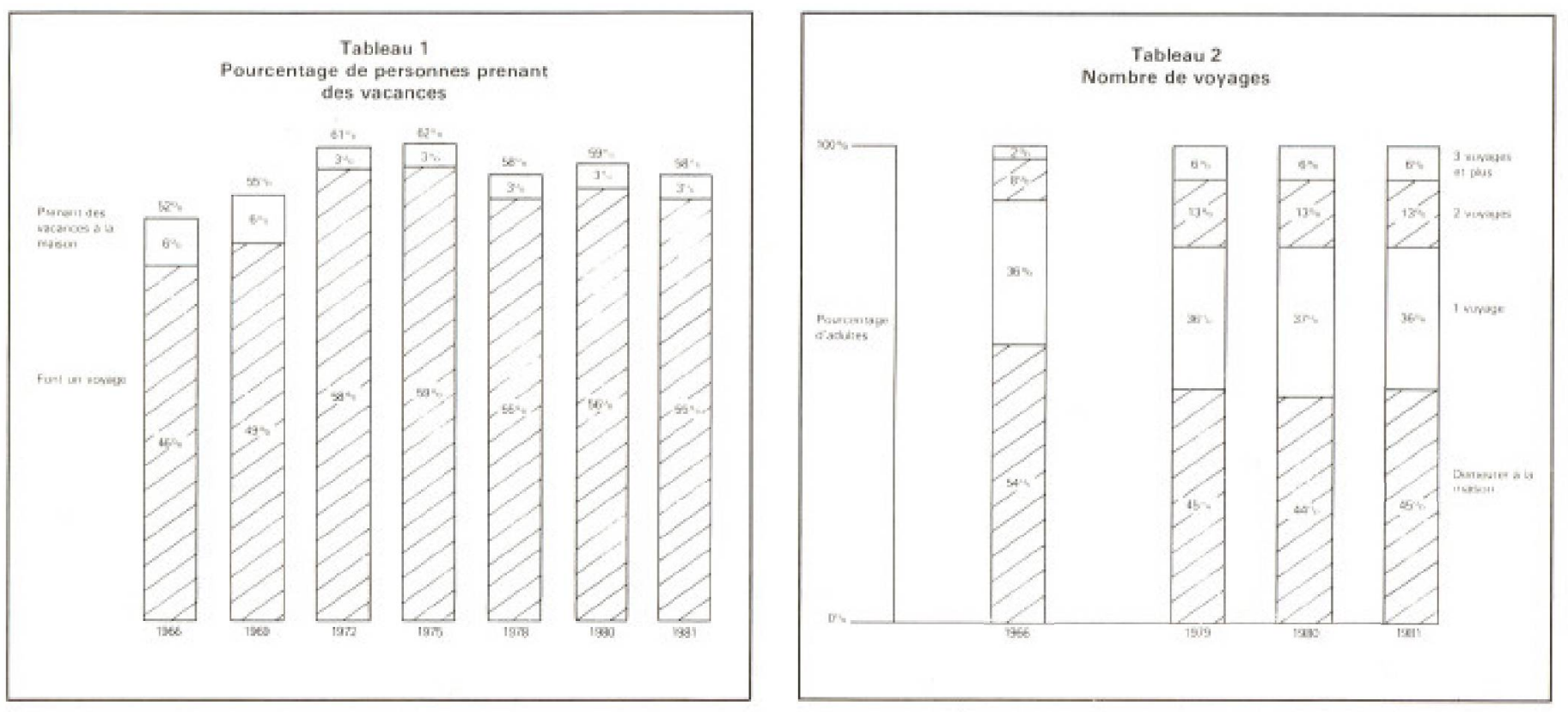



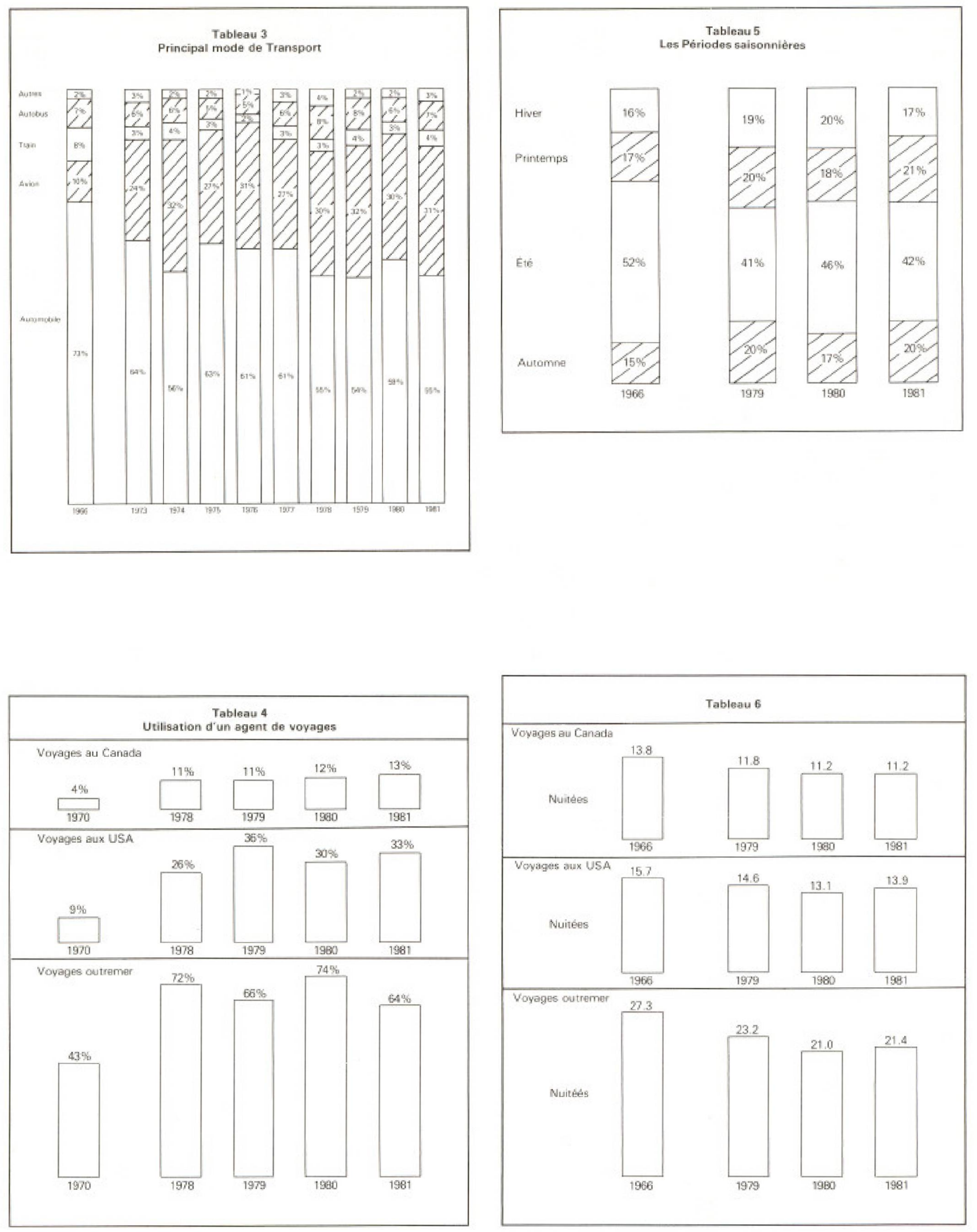
sens on peut confirmer certains faits. Résumons nos propos.

Même sil la crise de l'énergie n'a pas empêché les personnes de prendre des vacances, elle a cependant modifié sensiblement leurs habitudes. On a toute les raisons de croire que ce n'est pas là l'unique motif; bien d'autres raisons peuvent être responsables de ces changements: l'accroissement du revenu familial et l'augmentation du nombre de femmes sur le marché du travail comme le développement de nouveaux produits et de nouvelles destinations. Bref, I'interrelation de divers facteurs fait que les touristes ont modifié leurs habitudes de vacances au cours des vingt dernières années. C'est ainsi que, de plus en plus, on semble privilégier le fractionnement des vacances et, j'ai l'impression que cette tendance s'accroitra dans les années futures. Divers raisons mincitent a penser ainsi : pour n'en citer que quelques-unes, l'éventuelle popularité du condominium à temps partagé, l'accroissement des activités hivernales, les congés scolaires semestriels, etc... On remarque également qu'on a de plus en plus tendance à prendre des vacances en dehors de chez soi ce qui est attribuable sans doute à un revenu familial plus substantiel. Cette conséquence d'ailleurs influencera davantage l'ensemble des autres facteurs modifiant les habitudes de voyages. L'accroissement impressionnant des voyages par avion et par consequent de la plus grande popularité des agences de voyages ne sont certes par étrangers à ce facteur économique.

Vu globablement, au cours des ans, le touriste s'est transformé sensiblement et, de toute évidence, cette evolution se poursuivra. Ce r'est pas un phénomene stable, le touriste bouge constamment et souvent d'une façon imprévisible. Par contre, ce changement se poursuit subtilement et son évolution est relativement lente et semble obéir à des cycles de 10 ans. Ainsi, la décennie de 1960 à 1970 peut être qualifiée, en tout cas au Québec, comme une renaissance du tourisme et cette période correspond d'ailleurs à la révolution tranquille. Cet élan a été brusquement ébranlé par la crise de l'énergie et les années 1970 se caractérisent par une sorte de ressaisissement. Depuis les débuts des années 80 , on semble s'acheminer vers une décennie troisième vague oú les habitudes changent substantiellement. Peut-on prophétiser en supposant que les années 1990 favoriseront une seconde renaissance du tourisme. Dans cette perspective, il faudra s'y préparer.

II est pensable d'imaginer que l'évolution du phénomène touristique se poursuive au même rythme que celui de la société mais rien ne nous le confirme. Peut être

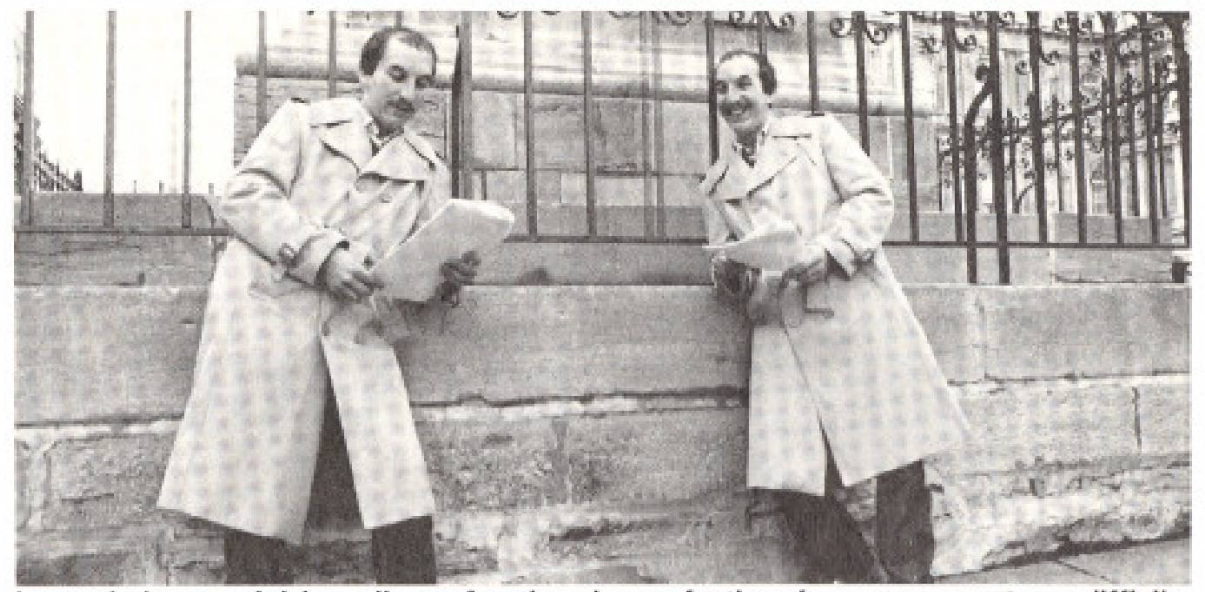

Les techniques prévisionnelles, même les plus perfectionnées, ne pourront que difficilement nous indiquer la voie â suivre à cause du phénomène lui-mème, c'est-â-dire que l'individu procede a un libre choix face à de multiples possibilités.

serons-nous à nouveau surpris pour finalement se rendre compte que les décisions prises ne sont pas nécessairement les bonnes. Les techniques prévisionnelles, même les plus perfectionnées, ne pourront que difficilement nous indiquer la voie à suivre à cause du phênomène luimême, c'est-à-dire que l'individu procède à un libre choix face à de multiples possibilités. Dans de telles conditions, peu de modèles prévisionnels fiables sont disponibles et ne le seront possiblement jamais.

Cependant, une étude du type de Travel Data, où l'on possède une information fondée sur les mêmes bases depuis près de 20 ans, constitue un outil intéressant en ce sens qu'il est possible de suiwre l'evolution du comportement des touristes. Par contre, bien que j'ai eu l'audace de généraliser quelque peu les données au cours du présent article, il r'en demeure pas moins que celles-ci sont restrictives. Rappelons-le, il s'agit dans le cas de Travel Data de touristes d'agrément canadiens. Un sondage á l'échelle canadienne, compte tenu de l'importance de l'échan. tillon, ne permet pas toujours de tirer des conclusions au niveau des provinces. II m'apparaitrait alors risque de conclure, ex cathedra, que mes propos s'appliquent sans nuance au Québec.

Par contre, à défaut de séries chronologiques stables touchant le Québec en particulier, il est permis de raisonner, avec prudence sans doute, à partir des outils disponibles.

Ceci étant dit, je ne peux m'empêcher de déplorer le manque de stabilité dans nos données statistiques. C'est là une lacune fondamentale puisqu'à partir de donnees statistiques fiables, de séries chronologiques stables, il est possible de planifier avec beaucoup plus de logique. Sans doute aurait-on dû, dès la création du ministère du Tourisme, de la Chasse et de la Pêche en 1964, amorcer une telle dèmarche au meilleur de notre connais: sance et respecter par la suite les bases statistiques de façon à pouvoir établir des comparaisons. Nous disposerions d'un indice touristique qui nous informerait sur les tendances avec un degré de fiabilité connu.

Par fiabilité, je n'entends pas nécessairement en valeur absolue, c'est-à-dire que cet indice nous donne des indications quantitatives à un moment donné, mais bien plus des tendances par rapport ă certaines variables de comportement. Qui plus est, ce genre d'indice peut bien être biaisé au départ, et ce n'est pas là une inquiétude, en autant qu'on maintienne la continuité du systeme. Par exemple, je faisais allusion précédemment a l'indice boursier; bien peu d'individus sont en mesure de comprendre la signification de l'indice Dow Jones qu'on nous présente chaque soir sur l'écran de TV et la plupart s'en balancent. Par contre, s'il y a hausse ou baisse, la signification a un tout autre sens et indique avec un certain degré le comportement des transactions dans le milieu des affaires.

Malgré cette lacune, il faut souligner que depuis quelques années, Statistique Canada a adopté une approche uniforme, tel que l'explique $\mathrm{P}$. Cluzeau dans son article. Même si on décèle en cours de route quelques lacunes, il m'apparaît nécessaire de songer à maintenir dorénavant l'uniformité des données afin de créer des séries permanentes dans le temps. Ceci n'empêche pas cependant d'améliorer la qualité des resultats (accroître la précision) tout en ayant la précaution de maintenir les mêmes bases comparatives.

Au terme de ces quelques réflexions, une chose est certaine: le tourisme évolue, Peut-être devrait-on maintenant se demander si nous, les responsables du tourisme, avons évolué?

\section{Note:}

I1I Ce growpe de travail existe toujours. 